\title{
Autoavaliação de médicos veterinários sobre sua competência clínica ao ingressarem em programas de residência
}

\author{
Gabriela Marin van der Broocke Campos ${ }^{1}$, Rafael Stedile ${ }^{2}$, Simone Tostes de Oliveira ${ }^{3}$, \\ Karynn Vieira Capilé ${ }^{4}$
}

${ }^{1}$ Graduanda da Universidade Federal do Paraná, Departamento de Medicina Veterinária. Curitiba-PR Brasil. E-mail: gmvdbc@hotmail.com

${ }^{2}$ Prof. Doutor em Medicina Veterinária, Conselho Regional de Medicina Veterinária do Paraná (CRMV-PR). Curitiba-PR Brasil.E-mail: stediler@gmail.com

${ }^{3}$ Professora da Universidade Federal do Paraná, Departamento de Medicina Veterinária. Curitiba-PR Brasil. E-mail: tostesimone@gmail.com

${ }^{4}$ Doutoranda da Universidade Federal Fluminense, Departamento Instituto de Ciências Biomédicas. Rio de Janeiro-RJ Brasil.E-mail: karynn.capile@gmail.com

*Autor para correspondência

RESUMO. O aumento do número de cursos de Medicina Veterinária em instituições de ensino superior no Brasil foi significativo na última década. Para o controle desta expansão é importante que se realizem avaliações de qualidade do ensino. O objetivo deste estudo foi avaliar a competência clínica de médicos veterinários, ao ingressarem em Programas de Residência no Brasil. Para tanto, um questionário de autoavaliação foi aplicado a residentes dos Programas de Residência em Medicina Veterinária de todas as regiões do país, sendo instruídos a responder de acordo com os conhecimentos adquiridos no período prévio à residência. Foram 90 questões divididas em habilidades clínicas referentes à procedimentos em geral e práticas em pequenos e em grandes animais. Cada habilidade deveria ser classificada em não adquirida, parcialmente adquirida ou plenamente adquirida. A porcentagem média de habilidades plenamente adquiridas foi de $57,4 \%$ para procedimentos em geral, 33,2\% para habilidades clínicas em pequenos animais e $11,7 \%$ para habilidades clínicas em grandes animais. A deficiência observada no aprendizado de forma geral pode decorrer da falta de treinamento clínico para os estudantes. Os resultados obtidos podem servir de guia tanto na graduação quanto nos programas de residência, visando a detecção das maiores deficiências e instituição de medidas corretivas.

Palavras chave: animais, graduação, habilidades, procedimentos, questionário

\section{Veterinarians self assessment on their clinical skills when getting into residency programs}

ABSTRACT. In the last decade, there was an upsurge in Veterinary Medicine
undergraduate programs in Brazil. To control this expansion, it is important to evaluate
the quality of teaching. This study had the goal of evaluating the clinical competence of
veterinarians as they start their residency. Therefore, a self-evaluation questionnaire was
applied to residents across all regions of the country. They were instructed to answer it
according to the knowledge they had prior to residency. The questionnaire consisted of 90
questions, divided between clinical skills related to general procedures and practices in
small and large animals. Each skill had to be classified as one of: not acquired, partially
acquired or fully acquired. The average percentage of skills fully acquired was $57.4 \%$ for
general procedures, $33.2 \%$ for clinical skills in small animals, $11.7 \%$ for large animals.
The knowledge gap observed may be caused by insufficient training on clinical treatment.
The results obtained in this study can be used as a guide for both undergraduate and 
residency programs, aiming to detect major areas for improvement to then apply corrective measures.

Keywords: animals, graduation, skills, procedures, questionnaire

\section{Introdução}

Na última década, o número de instituições de ensino superior (IES) que oferecem curso de graduação em Medicina Veterinária (MV) no Brasil aumentou significativamente. Em 2010, o Ministério da Educação (MEC) informava a existência de 155 IES com cursos de MV (Borges et al., 2013). Atualmente há no Brasil 253 cursos de MV em situação ativa conforme os dados disponíveis no sítio do MEC na internet (BRASIL, 2016). Para que exista algum tipo de controle qualitativo desta expansão é importante que se possa avaliar a qualidade do ensino, detectar carências de aprendizagem e desenvolver estratégias didáticas capazes de suprir estas carências (Sobrinho, 2010). Neste âmbito, a autoavaliação tem sido considerada um importante meio de acessar o grau de conhecimento dos estudantes. Boud (1999) define autoavaliação como o envolvimento do estudante na identificação de padrões ou critérios para avaliar seu trabalho e no julgamento a respeito destes critérios e padrões. Atribui-se à técnica de autoavaliação um efeito auto-reflexivo nos estudantes que ao se autoavaliarem tornam-se mais perceptivos e atentos as suas próprias competências e limitações e, com isto, tendem a se empenhar mais em superar as dificuldades identificadas (Dearnley and Meddings, 2007).

Apesar de haver consenso na literatura científica quanto à relevância de avaliações que forneçam informações sobre a competência clínica adquirida pelos médicos veterinários durante sua formação; avaliações deste tipo ainda são incipientes e há poucos estudos a respeito de atributos específicos, como habilidades clínicas, na literatura médico veterinária (Rhind, 2006). Considerando a importância de se identificar perfis de aprendizado durante as etapas da formação profissional medicina veterinária, o objetivo deste estudo foi investigar a competência clínica, por meio de autoavaliação, dos médicos veterinários ingressantes em Programas de Residência no Brasil.

\section{Material e Métodos}

Para avaliar a aptidão e segurança referentes às habilidades clínicas adquiridas durante o curso de graduação em medicina veterinária e também durante experiência profissional prévia ao ingresso na residência, foi aplicado um questionário de autoavaliação a residentes dos Programas de Residência em Medicina Veterinária no Brasil.

O questionário foi composto de 90 questões sobre a aptidão em habilidades clínicas do respondente ao ingressar no programa de residência. Foi pedido a cada respondente que considerasse apenas o período prévio ao seu ingresso na residência, de forma que $o$ conhecimento adquirido durante a residência não fosse levado em conta. Os itens estão listados na Tabela 1 e eram referentes a: procedimentos em geral (6), práticas em pequenos animais (49) e práticas em grandes animais (35). Em cada item, o respondente deveria classificar suas habilidades como: não adquirida (não se considerava apto a realizar o procedimento), parcialmente adquirida (apresentava o conhecimento da técnica, mas possuía dúvidas de execução ou insegurança em realizá-la) ou plenamente adquirida (apresentava conhecimento teórico e estava apto a realizá-la com segurança). Além disso, foi questionado se métodos alternativos ao uso de animais vivos auxiliariam no aprendizado de habilidades clínicas. Foi incluído também espaço para possíveis comentários gerais. O questionário foi elaborado de forma que seu respondente $\mathrm{e}$ instituição permanecessem anônimos, limitandose a perguntar sobre: a região do país na qual se incluía seu Programa de Residência; a área de atuação ou espécies com as quais trabalha; e se já possuía experiência profissional prévia à residência.

Foram listadas as universidades brasileiras com Programas de Residência em Medicina Veterinária reconhecidos pelo Conselho Federal de Medicina Veterinária e/ou pelo MEC. A partir desta lista, buscou-se o contato via telefone com os coordenadores de programas de residência das 32 universidades cadastradas. Nas conversas telefônicas houve uma breve explanação das propostas da pesquisa e solicitação de e-mail para encaminhamento do link do questionário online a ser destinado aos residentes do programa. Dessa forma, o questionário teve como alvo todas as regiões do país (Sul, Sudeste, Centro-oeste, Norte 
e Nordeste). O questionário foi elaborado de forma a ser respondido anonimamente, por meio do Google forms ${ }^{\circledR}$, um recurso que permite a elaboração e preenchimento de formulários online. $\mathrm{O}$ prazo oferecido para o preenchimento do questionário por parte dos residentes foi de três meses.
Para análise estatística, foram utilizados o teste $\mathrm{T}$ e a Análise de Variância (ANOVA), seguida do teste de Tukey. Considerou-se estatisticamente significativo uma confiança de 95\% $(\mathrm{P}=0,05)$. Os dados foram apresentados como média e desvio-padrão.

Tabela 1. Itens auto-avaliados por médicos veterinários, ao ingressarem em programas de Residência em Medicina Veterinária, sobre suas habilidades clínicas.

\begin{tabular}{|c|c|}
\hline & Itens avaliados \\
\hline Gerais & $\begin{array}{l}\text { Comunicação veterinário-cliente; raspado de pele; biópsia por aspiração por agulha fina; } \\
\text { teste de produção lacrimal; preparação asséptica da pele; sutura (procedimentos } \\
\text { simples/"ambulatorial") }\end{array}$ \\
\hline Em cães e gatos & $\begin{array}{l}\text { Exame físico geral (de rotina); cardiovascular/respiratório; neuromuscular/ ortopédico; } \\
\text { oftálmico; digestório; odontológico; urinário/reprodutor; dermatológico (pele e anexos); } \\
\text { comportamental; atendimento de emergência; contenção física de cães; contenção física de } \\
\text { gatos; coleta de sangue em cães; coleta de sangue em gatos; administração oral de } \\
\text { medicamentos; aplicação de medicamentos intramuscular e subcutâneo; colocação de cateter } \\
\text { intravenoso; colocação de cateter intra-ósseo; coleta de medula óssea; aplicação tópica } \\
\text { oftálmica; exame otológico; sondagem urinária (cães machos); sondagem urinária (gatos } \\
\text { machos); sondagem urinária em cadelas com visualização direta (espéculo vaginal); } \\
\text { sondagem urinária em cadelas com técnica tátil; exame retal digital (cães machos); limpeza } \\
\text { e canulação de sacos anais; corte de unhas; enema; intubação traqueal; sondagem orogástrica } \\
\text { e lavagem gástrica; intubação nasogástrica em gatos; colocação de tubo de faringostomia; } \\
\text { colocação de tubo percutâneo de gastrostomia; lavado traqueal; lavado broncoalveolar; } \\
\text { colocação de tubo torácico (dreno torácico); toracocentese; abdominocentese; cistocentese; } \\
\text { pericardiocentese; artrocentese; coleta de sêmen e inseminação artifical; exame vaginal e } \\
\text { coleta de amostras vaginais (swab); coleta de liquido cérebro espinhal (cisterna magna); } \\
\text { coleta de líquido cerebroespinhal (lombar)/punção lombar; tempo de sangramento da } \\
\text { mucosa oral; avaliação de dor; imobilizações/bandagens. }\end{array}$ \\
\hline $\begin{array}{l}\text { Em grandes } \\
\text { animais }\end{array}$ & $\begin{array}{l}\text { Exame físico geral (de rotina); cardiovascular/respiratório; locomotor/ } \\
\text { neurológico/ortopédico; oftálmico; digestório de ruminantes; digestório de equinos/abdome } \\
\text { agudo (cólica); urinário; reprodutor; dermatológico (pele e anexos). Contenção de equinos; } \\
\text { contenção de bovinos; contenção de caprinos; contenção de ovinos; contenção de suínos; } \\
\text { coleta de sangue; sondagem urinária em vaca; sondagem urinária em égua; sondagem } \\
\text { urinária em ovelhas, cabras e porcas; sondagem urinária em cavalos (machos); lavado } \\
\text { transtraqueal; intubação traqueal em equinos; administração de medicamentos orais; } \\
\text { administração de medicamentos parenterais; sondagem nasogástrica em equinos; sondagem } \\
\text { ruminal; abdominocentese; palpação retal em bovinos; palpação retal em equinos; } \\
\text { artrocentese; coleta de sêmen em ruminantes; coleta de sêmen em equinos; bloqueios } \\
\text { anestésicos regionais; lavagem uterina; remoção de ferraduras; imobilizações/bandagens. }\end{array}$ \\
\hline
\end{tabular}

\section{Resultados}

Um total de 147 questionários foi respondido, com participação das cinco regiões do Brasil. A média do número de habilidades clínicas plenamente adquiridas, referente aos 90 itens abordados, foi de $23 \pm 12$ habilidades por questionário respondido, variando a amplitude de 1 a 65 habilidades. Das 90 habilidades clínicas presentes no questionário, 6 se referiam a procedimentos em geral, 49 se referiam a pequenos animais e 35 a grandes animais. A porcentagem média de habilidades plenamente adquiridas foi de $57,4 \% \quad(3,4 / 6)$ para procedimentos em geral, $33,2 \%(15,9 / 49)$ para habilidades clínicas em pequenos animais e $11,7 \%(4,1 / 35)$ para habilidades clínicas em grandes animais. As comparações das habilidades estão apresentadas na Tabela 2.

Dos 90 procedimentos avaliados no questionário, os 15 procedimentos com mais respondentes em média se considerando aptos e os 15 com mais respondentes se considerando não aptos estão listados na Tabela 3. 
Tabela 2. Autoavaliação, de 147 médicos veterinários ingressantes em programas de Residência, em relação às suas habilidades clínicas, na área de pequenos ou grandes animais, e habilidades gerais. Média do número de respondentes \pm desvio padrão. Entre parênteses, a porcentagem do número de respondentes em relação ao total (147).

\begin{tabular}{lcccc}
\hline Grupo das habilidades & $\begin{array}{c}\text { Plenamente } \\
\text { adquiridas }\end{array}$ & $\begin{array}{c}\text { Parcialmente } \\
\text { adquiridas }\end{array}$ & $\begin{array}{c}\text { Não } \\
\text { adquiridas }\end{array}$ & $\begin{array}{c}\text { Não } \\
\text { responderam }\end{array}$ \\
\hline Gerais & $84.33 \pm 17.58^{\mathrm{a}}$ & $48.83 \pm 11.35^{\mathrm{a}}$ & $13.5 \pm 11.14^{\mathrm{a}}$ & $0.0^{\mathrm{a}}$ \\
& $(57.36 \%)$ & $(33.21 \%)$ & $(9.18 \%)$ & $(0.0 \%)$ \\
Em Pequenos Animais & $47.83 \pm 38.80^{\mathrm{b}}$ & $51.69 \pm 21.28^{\mathrm{a}}$ & $46.57 \pm 38.91^{\mathrm{a}}$ & $0.59 \pm 0.86^{\mathrm{a}}$ \\
& $(33.21 \%)$ & $(35.16 \%)$ & $(31.68 \%)$ & $(0.40 \%)$ \\
Em Grandes Animais & $17.25 \pm 13.99^{\mathrm{c}}$ & $43.8 \pm 14.95^{\mathrm{a}}$ & $81.92 \pm 23.62^{\mathrm{b}}$ & $4.11 \pm 0.63^{\mathrm{b}}$ \\
& $(11.73 \%)$ & $(29.79 \%)$ & $(55.66 \%)$ & $(2.79 \%)$ \\
Todas & $38.37 \pm 35.65$ & $48.43 \pm 18.74$ & $58.07 \pm 38.28$ & $1.92 \pm 1.91$ \\
\hline
\end{tabular}

Os valores identificados com a mesma letra na mesma coluna não apresentam diferença significativa.

Tabela 3. Lista dos 15 procedimentos com maior e menor número de respondentes se considerando aptos para realizá-los, e com maior número se considerando não aptos. $\mathrm{PA}=\mathrm{em}$ pequenos animais, GA=em grandes animais; Geral=procedimentos em geral.

\begin{tabular}{|c|c|c|c|}
\hline $\begin{array}{l}\text { Procedimentos com mais respondentes em } \\
\text { média se considerando plenamente aptos }\end{array}$ & $\%$ & $\begin{array}{l}\text { Procedimentos com mais respondentes em } \\
\text { média se considerando NÃO aptos }\end{array}$ & $\%$ \\
\hline Administração oral de medicamentos (PA) & 90,5 & Artrocentese (GA) & 82,3 \\
\hline $\begin{array}{l}\text { Aplicação de medicamentos via subcutânea e } \\
\text { intramuscular (PA) }\end{array}$ & 89,1 & Remoção de ferraduras (GA) & 81,6 \\
\hline Aplicação tópica oftálmica (PA) & 85,7 & $\begin{array}{l}\text { Sondagem urinaria em ovelhas, cabras e } \\
\text { porcas }\end{array}$ & 80,9 \\
\hline Preparação asséptica da pele (Geral) & 80,3 & $\begin{array}{l}\text { Colocação de tubo percutâneo de } \\
\text { gastrostomia (PA) }\end{array}$ & 78,2 \\
\hline Exame físico geral de rotina (PA) & 75,5 & [Sondagem urinária em vaca & 77,5 \\
\hline Contenção de cães & 74,8 & Lavado transtraqueal (GA) & 77,5 \\
\hline Sondagem urinária de cães machos & 70,7 & Sondagem urinária em égua & 76,9 \\
\hline Corte de unhas (PA) & 69,4 & Lavado broncoalveolar (PA) & 76,2 \\
\hline Coleta de sangue em cães & 68,0 & Pericardiocentese (PA) & 76,2 \\
\hline Colocação de cateter intravenoso (PA) & 67,3 & Artrocentese (PA) & 74,1 \\
\hline Contenção de gatos & 59,8 & $\begin{array}{l}\text { Coleta de liquido cerebroespinhal (cisterna } \\
\text { magna) (PA) }\end{array}$ & 72,8 \\
\hline Coleta de sangue em gatos & 59,2 & Lavagem uterina (GA) & 72,8 \\
\hline $\begin{array}{l}\text { Sutura em procedimentos } \\
\text { simples/"ambulatorial" (Geral) }\end{array}$ & 57,8 & Lavado traqueal (PA) & 71,4 \\
\hline Raspado de pele (GERAL) & 55,8 & Coleta de sêmen em equinos & 71,4 \\
\hline Comunicação veterinário-cliente (Geral) & 54,4 & $\begin{array}{l}\text { Coleta de líquido cérebro espinhal/punção } \\
\text { lombar (PA) }\end{array}$ & 70,7 \\
\hline
\end{tabular}


Foram analisados também os 15 procedimentos com menos respondentes se considerando plenamente aptos (dados não mostrados em tabela). Destes, os procedimentos citados foram os mesmos listados como procedimentos com mais respondentes se considerando não aptos, com exceção de 4 procedimentos (coleta de medula óssea em cães e gatos, exame do sistema urinário em grandes animais, colocação de dreno torácico em cães e gatos, exame locomotor/neurológico/ortopédico em grandes animais)

Do total de respondentes, $85(57,8 \%)$ não apresentavam experiência profissional prévia à residência e $62(42,1 \%)$ apresentavam. No grupo sem experiência profissional prévia, a média do número de habilidades plenamente adquiridas foi $22 / 90(24,5 \%)$, enquanto que no grupo com experiência prévia foi de $25 / 90$ (27,8\%). Não houve diferença estatística entre apresentar ou não experiência profissional prévia e o número de habilidades plenamente adquiridas $(\mathrm{p}=0,21)$.

A área de atuação para a qual o residente ingressou no Programa de Residência foi reagrupada em grupos maiores, sendo estes: Anestesiologia, Pequenos Animais, Grandes Animais, Animais Selvagens, Patologia, Patologia Clínica, Diagnóstico por Imagem, Medicina do Coletivo, Medicina Veterinária Preventiva e Outros (áreas com apenas 1 ou 2 respondentes, ou não especificadas).

Em relação à área de ingresso na residência, as maiores médias das habilidades plenamente adquiridas foram das especialidades de Diagnóstico por Imagem, Animais Selvagens e Anestesiologia, e asmenores médias foram das especialidades de Patologia, Patologia Clínica e Medicina do Coletivo. As médias de habilidades plenamente adquiridas referentes às diferentes especialidades estão descritas na Tabela 4.

Tabela 4. Média das habilidades clínicas plenamente adquiridas, de acordo com a autoavaliação dos médicos veterinários ao ingressarem no Programa de Residência em Medicina Veterinária, considerando a área de ingresso.

\begin{tabular}{lc}
\hline $\begin{array}{l}\text { Grupo de especialidade na qual o residente } \\
\text { ingressou no programa }^{1}\end{array}$ & $\begin{array}{c}\text { Média de habilidades plenamente adquiridas } \\
(90 \text { itens })\end{array}$ \\
\hline Diagnóstico por imagem & $29,87 \pm 13,87(33,2 \%)$ \\
Animais selvagens & $29,14 \pm 15,91(32,4 \%)$ \\
Anestesiologia & $28,93 \pm 8,16(32,1 \%)$ \\
Grandes animais & $26,8 \pm 14,71(29,7 \%)$ \\
Pequenos animais & $25,29 \pm 10,58(28,1 \%)$ \\
Preventivo & $23,33 \pm 15,85(25,9 \%)$ \\
Outros & $20,88 \pm 6,82(23,2 \%)$ \\
Patologia & $15,07 \pm 10,68(16,7 \%)$ \\
Patologia clínica & $14,64 \pm 10,12(16,3 \%)$ \\
Medicina do coletivo & $14 \pm 7,69(15,6 \%)$ \\
\hline
\end{tabular}

${ }^{1}$ As especialidades foram agrupadas em grupos maiores

Ao serem comparadas as áreas, considerando as habilidades plenamente adquiridas, observouse diferença significativa $(<0,05 \%)$ entre Anestesiologia e Patologia, entre Anestesiologia e Patologia Clínica e entre Pequenos Animais e Patologia Clínica. A figura 1 mostra como as habilidades plenamente adquiridas estão distribuídas em cada área, em relação aos itens avaliados (gerais, grandes animais e pequenos animais). Dos que se posicionaram a favor, os argumentos utilizados incluíram evitar-se o sofrimento desnecessário dos animais, garantirem bem-estar animal, não constranger os estudantes e ser uma boa opção para o primeiro contato com procedimentos clínicos. Dentre os que se posicionaram contra, os principais comentários incluíram o fato de se tratar de uma forma menos realista ao se comparar com o aprendizado a partir de um paciente real e trazer maior insegurança na execução futura do procedimento 
em um animal vivo. Em relação às respostas da autoavaliação, não houve diferença significativa no aprendizado entre quem acreditava e quem não acredita na eficácia dos métodos alternativos.

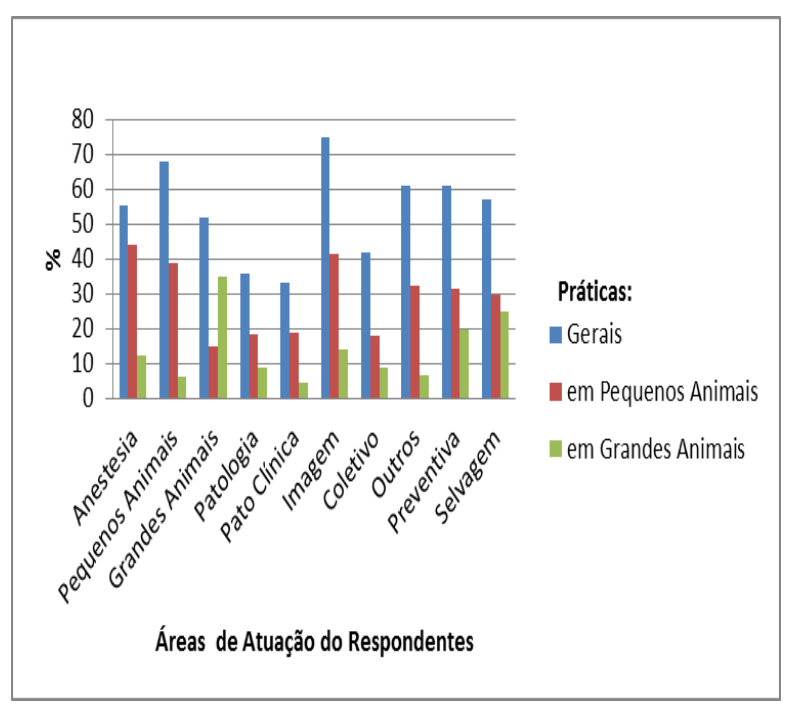

Figura 1. Habilidades plenamente adquiridas (procedimentos em geral, práticas em pequenos animais e práticas em grandes animais), com respondentes distribuídos em cada área de ingresso nos Programas de Residência em Medicina Veterinária.

Quando questionados a respeito da utilização de métodos alternativos ao uso de animais vivos no aprendizado de habilidades clínicas, 97 (66\%) responderam acreditar que estes métodos podem auxiliar no ensino, $46(31 \%)$ responderam não acreditar e 5 pessoas (3\%) não se posicionaram.

\section{Discussão}

A avaliação propicia reformas educacionais capazes de gerar mudanças metodológicas e curriculares relativas ao ensino, incluindo conceitos e práticas de formação, modelos institucionais, estruturas de poder, políticas educacionais, prioridades de pesquisa e noções de responsabilidade social (Sobrinho, 2010). Desta forma, nos colocamos diante de questões sobre como avaliar a qualidade do ensino/aprendizado por meio do conhecimento adquirido pelos alunos, o que parece ainda mais complexo quando se trata de habilidades práticas. Os resultados aqui apresentados podem servir de guia tanto para a estruturação curricular dos cursos de graduação quanto para os programas de residência, para se perceber onde estão as maiores deficiências e corrigi-las.

A escolha de residentes para a aplicação do questionário se deve ao fato de serem geralmente estudantes engajados com a Universidade durante a graduação, com perfil crítico, estudiosos e curiosos, possibilitando avaliação fidedigna do ensino nesta etapa acadêmica.

Não houve diferença estatística entre apresentar ou não experiência profissional prévia e o número de habilidades plenamente adquiridas, o que sugere que um ou dois anos de trabalho não acadêmico após a formação não resultaram em maior aquisição de habilidades clínicas. Isto demonstra que a atuação no mercado de trabalho por si só não possibilita o desenvolvimento de mais habilidades, pois o médico veterinário aparentemente continua inseguro para a realização de tais procedimentos, se considerando não apto ou parcialmente apto quanto à determinadas habilidades clínicas. Portanto, cabe a reflexão sobre em que momento estas habilidades deveriam ser desenvolvidas, pois algumas delas são bastante específicas e não fazem parte de procedimentos rotineiros e são mais invasivos, e outras são procedimentos gerais, como exame físico do sistema urinário em grandes animais e exame locomotor/neurológico /ortopédico. A residência é uma excelente oportunidade para aquisição destas habilidades, visto a grande casuística e a oportunidade de se treinar procedimentos específicos de cada área, de forma supervisionada. Porém não se deve negligenciar o ensino destas durante a graduação, visto a diferença de (poucas) vagas ofertadas na residência e a (grande) procura.

Considerando as médias obtidas de habilidades plenamente adquiridas em relação à área de residência, visto que ao responder o questionário o respondente deveria considerar o momento do seu ingresso, não considerando qualquer aprendizado durante a residência, este dado pode apenas sugerir uma tendência dos ingressantes nestes grupos de terem se interessado mais por estes procedimentos durante a graduação, em aula ou mesmo em estágios, visto imaginarem que na área de atuação tal conhecimento seria necessário, portanto estariam mais disposto em buscá-lo. Os grupos com menores médias podem não ter tido interesse ou não ter se dedicado a alguns tópicos, provavelmente apresentando outro foco de aprendizado durante a graduação.

Cursos de áreas da saúde que exigem o desenvolvimento de habilidades práticas além da aquisição de conhecimento teórico precisam garantir aos alunos oportunidades adequadas de 
treinamento prático, caso contrário, as habilidades clínicas podem não ser efetivamente desenvolvidas. De acordo com os dados obtidos nesta pesquisa, existe uma deficiência no aprendizado de forma geral. Talvez isto se deva à falta de oportunidade de treinamento clínico para os estudantes, uma vez que debates éticos e questões relativas ao bem-estar animal têm desencorajado professores e alunos a realizar treinamento de habilidades clínicas em animais (Capilé et al., 2015). Neste sentido, o uso de métodos alternativos poderia ser uma forma de solucionar parte das deficiências relatadas pelos médicos veterinários, visto que muitas das habilidades listadas por eles como não adquiridas referem-se a procedimentos invasivos. Para outros procedimentos não invasivos, como exame neurológico/ortopédico/locomotor em grandes animais e exame do sistema urinário em grandes animais, a informação para os professores de que muitos alunos não se sentem preparados para fazê-lo pode ser o ponto de partida para que uma maior atenção seja dada em relação a este aprendizado. A lista, aqui apresentada, com a autopercepção dos estudantes em relação às habilidades clínicas auxilia na identificação de quais procedimentos devem ser prioritariamente ensinados. Sugere-seque uma escala de prioridades seja elaborada de forma específica levando-se em consideração as particularidades de cada contexto: graduação, residência ou outros cursos de especialização, levando-se em conta também a área da medicina veterinária na qual cada grupo de aprendizes pretende atuar.

Outro fator que pode desencadear índices insatisfatórios de aprendizado é a estrutura curricular. Estudos referentes a estratégias de aprendizagem apontam que o tipo de motivação é fundamental para a efetivação do conhecimento. Quando o estudante não tem participação na escolha do conteúdo a ser estudado e não há relação direta entre o conteúdo e algum problema concreto e real identificado por ele mesmo, a tendência é que os alunos adquiram um conhecimento suficiente apenas para o cumprimento de metas curriculares circunstanciais. Currículos centrados em avaliações ou obtenção de conceitos e não na resolução de problemas significativos para os alunos, fazem com que estes estudem para obter aprovação e não para tornaram-se aptos a resolver problemas pertinentes a sua profissão (Neame and Powis, 1981, Slattery, 2012). Conhecimentos cuja utilidade é temporária tendem a não se fixar, o que explicaria o fato de os Médicos Veterinários ingressantes em programas de residência não dominarem certos conteúdos estudados durante a graduação. Métodos como o de aprendizado baseado na resolução de problemas $(P B L)$, o aprendizado independente $(I L)$, e que envolvem sistemas mais interativos, que integram múltiplos recursos de mídia e ambientais como e-learning e blended learning têm mostrado melhores resultados de aprendizagem (Butler and Winne, 1995, Wong, 2013). Entretanto, no Brasil, grande parte dos cursos de educação superior ainda segue metodologias de ensino tradicionais cujo papel do aluno é passivo. Com relação ao sistema de avaliação utilizado, ainda que alguns autores apontem a possibilidade de falta de acurácia no sistema de autoavaliação (Boud, 1999, Hildebrand et al., 2009), as deficiências identificadas pelos médicos veterinários indicam alguma inadequação metodológica nos cursos de graduação em Medicina Veterinária brasileiros, seja em relação ao aprendizado das habilidades clínicas em si ou em relação a outros aspectos educacionais da formação profissional do Médico Veterinário.

\section{Conclusão}

Considerando os resultados e hipóteses aqui apresentados, entende-se que as causas da deficiência de aprendizado são multifatoriais e é importante que sejam conduzidas mais investigações a este respeito. Como o presente estudo quantifica e qualifica falhas existentes no ensino em relação a habilidades clínicas na Medicina Veterinária, pode servir de ferramenta para a tomada de medidas de correção das deficiências observadas, tanto na graduação quanto nos Programas de Residência em Medicina Veterinária. Serve, portanto, de guia para a identificação das prioridades a serem aprimoradas no ensino.

\section{Referências bibliográficas}

Borges, T. D., Sans, E. C. O., Braga, J. S., Machado, M. F. \& Molento, C. F. M. (2013). Ensino de bem-estar e dor animal em cursos de medicina veterinária no Brasil. Arquivo Brasileiro de Medicina Veterinaria $e$ Zootecnia, 65, 29-36.

Boud, D. (1999). Avoiding the traps: seeking good practice in the use of self assessment and reflection in professional courses. Social Work Education, 18, 121-132. 
Butler, D. L. \& Winne, P. H. (1995). Feedback and self-regulated learning: A theoretical synthesis. Review of Educational Research, $65,245-281$.

Capilé, K. V., Campos, G. M. B., Stedile, R. \& Oliveira, S. T. (2015). Canine prostate palpation simulator as a teaching tool in veterinary education. Journal of Veterinary Medical Education, 42, 146-150.

Dearnley, C. A. \& Meddings, F. S. (2007). Student self-assessment and its impact on learning-A pilot study. Nurse Education Today, 27, 333-340.

Hildebrand, C., Trowbridge, E., Roach, M. A., Sullivan, A. G., Broman, A. T. \& Vogelman, B. (2009). Resident self-assessment and selfreflection: University of WisconsinMadison's five-year study. Journal of General Internal Medicine, 24, 361-365.

Neame, R. L. \& Powis, D. A. (1981). Toward independent learning: curricular design for assisting students to learn how to learn. Academic Medicine, 56, 886-93.

Rhind, S. M. (2006). Competence at graduation: implications for assessment. Journal of Veterinary Medical Education, 33, 172-175.
Slattery, P. (2012). Curriculum development in the postmodern era: Teaching and learning in an age of accountability. Routledge, New York.

Sobrinho, J. D. (2010). Avaliação e transformações da educação superior brasileira (1995-2009): do provão ao SINAES. Revista da Avaliação da Educação Superior, 15, 195-224.

Wong, L. (2013). Developing independent learning skills for postgraduate students through blended learning environment. Journal of Cases on Information Technology, $15,36-50$.

\section{Article History:}

Received 26 July 2016

Accepted 15 August 2016

Available on line 7 September 2016

License information: This is an open-access article distributed under the terms of the Creative Commons Attribution License, which permits unrestricted use, distribution, and reproduction in any medium, provided the original work is properly cite. 